\title{
Pre-pregnancy fried food consumption and the risk of gestational diabetes mellitus: a prospective cohort study
}

\author{
Wei Bao • Deirdre K. Tobias • Sjurdur F. Olsen • \\ Cuilin Zhang
}

Received: 6 June 2014 / Accepted: 29 August 2014 / Published online: 11 October 2014

(C) Springer-Verlag (outside the USA) 2014

\begin{abstract}
Aims/hypothesis Fried foods are frequently consumed in Western countries. However, the health effects of frequent fried food consumption in humans are not well understood. We aimed to prospectively examine the association between pre-pregnancy fried food consumption and risk of incident gestational diabetes mellitus (GDM).

Methods We included 21,079 singleton pregnancies from 15,027 women in the Nurses' Health Study II cohort. Since 1991 and every 4 years thereafter, we collected diet information, including consumption of fried foods at home and away from home, using a validated food frequency questionnaire. We used generalised estimating equations with log-binomial models to estimate the RRs and 95\% CIs.

Results We documented 847 incident GDM pregnancies during 10 years of follow-up. After adjustment for age, parity, dietary and non-dietary factors, the RRs (95\% CIs) of GDM
\end{abstract}

Electronic supplementary material The online version of this article (doi:10.1007/s00125-014-3382-x) contains peer-reviewed but unedited supplementary material, which is available to authorised users.

W. Bao $\cdot$ C. Zhang $(\bowtie)$

Epidemiology Branch, Division of Intramural Population Health

Research, Eunice Kennedy Shriver National Institute of Child Health

and Human Development, National Institutes of Health, 6100

Executive Blvd, Rockville, MD 20852, USA

e-mail: zhangcu@mail.nih.gov

D. K. Tobias

Department of Nutrition, Harvard School of Public Health,

Boston, MA, USA

D. K. Tobias

Division of Preventive Medicine, Department of Medicine, Brigham and Women's Hospital and Harvard Medical School,

Boston, MA, USA

S. F. Olsen

Centre for Fetal Programming, Department of Epidemiology

Research, Statens Serum Institut, Copenhagen, Denmark among women who consumed total fried foods $1-3,4-6$ and $\geq 7$ times/week, compared with those who consumed it less than once/week, were $1.13(0.97,1.32), 1.31(1.08,1.59)$ and $2.18(1.53,3.09)$, respectively ( $p$ for trend $<0.001)$. The association persisted after further adjustment for BMI ( $p$ for trend $=0.01)$. When analysed separately, we found a significant association of GDM with fried food consumption away from home, but not with fried food consumption at home. Conclusions/interpretation Frequent fried food consumption, particularly away from home, was significantly associated with a greater risk of incident GDM. Our study indicates potential benefits of limiting fried food consumption in the prevention of GDM in women of reproductive age.

Keywords Diet . Fried food - Gestational diabetes mellitus

$\begin{array}{ll}\text { Abbreviations } \\ \text { AHEI-2010 } & \text { Alternate Healthy Eating Index 2010 } \\ \text { FFQ } & \text { Food frequency questionnaire } \\ \text { GDM } & \text { Gestational diabetes mellitus } \\ \text { GEE } & \text { Generalised estimating equations } \\ \text { MET } & \text { Metabolic equivalent } \\ \text { NHS } & \text { Nurses' Health Study }\end{array}$

\section{Introduction}

Fried foods are frequently consumed in Western countries [1, 2]. With globalisation, the number of food industries and outlets that produce and sell fried food, particularly fast food, is increasing rapidly around the world [2-4]. Frying is a complex cooking process that modifies both the foods and the frying medium. It reduces water content, increases energy density, changes fatty acid composition and deteriorates frying 
oils, especially when reused, through the processes of oxidation and hydrogenation [5]. Frying also makes food crunchy and aromatic and improves food palatability, which may in turn lead to excess intake [6]. Recently, frequent consumption of fried foods has been linked to a higher risk of being overweight and obesity in two Mediterranean cohorts $[6,7]$. However, there are few prospective epidemiological studies examining the association of fried food consumption with other health outcomes.

Gestational diabetes mellitus (GDM) is a common pregnancy complication characterised by glucose intolerance with onset or first recognition during pregnancy [8]. GDM is not only associated with short-term perinatal outcomes [9], but is also related to long-term metabolic risk in both mothers and their offspring $[8,10,11]$. Thus, it is crucial to identify modifiable risk factors that may contribute to the prevention of GDM. Previous studies have identified a number of dietary and other lifestyle factors related to GDM risk [12]. Fried foods contain some deleterious substances, e.g., oil degradation products, which may have adverse effects on glucose homeostasis. The association between fried food consumption and GDM risk, however, remains unknown. In this prospective cohort study, we examined the association between prepregnancy fried food consumption, both at home and away from home, and the risk of subsequent GDM.

\section{Methods}

Study population The Nurses' Health Study II (NHS II) is an ongoing prospective cohort study of 116,671 female nurses aged 25-44 years at study inception in 1989 [13]. The participants received a biennial questionnaire regarding disease outcomes and lifestyle behaviours, such as smoking status and medication use. Follow-up for each questionnaire cycle was greater than $90 \%$ [14]. This study has been approved by the Partners Human Research Committee (Boston, MA, USA), with the consent of participants implied by the return of the questionnaires.

We included NHS II participants in this analysis if they reported at least one singleton pregnancy lasting longer than 6 months (electronic supplementary material [ESM] Fig. 1). The 1991 questionnaire was the first time dietary information was collected. Thus, we set this year as the baseline for this analysis and we only included pregnancies after the return of the 1991 questionnaire. The 2001 questionnaire was the last time GDM was ascertained, since the majority of NHS II participants had passed reproductive age by then; therefore, follow-up was through the return of the 2001 questionnaire. Individual pregnancies were eligible if there was no GDM reported in a previous pregnancy, or a prior diagnosis of type 2 diabetes mellitus, cardiovascular disease or cancer. We excluded pregnancies if the participant did not return at least one pre-pregnancy food frequency questionnaire (FFQ), left more than 70 FFQ items blank or reported unrealistic total energy intake $(<2,510$ or $>14,644 \mathrm{~kJ} /$ day, which is equivalent to $<600$ or $>3,500 \mathrm{kcal} /$ day). Women with GDM in a previous pregnancy were excluded because they may change their diet and lifestyle during the next pregnancy to prevent recurrent GDM.

Exposure assessment Beginning in 1991 and every 4 years thereafter, we asked the participants to report their usual food intake over the previous year using a previously validated FFQ [15-17]. For fried food consumption, we asked the participants 'How often do you eat fried food away from home (e.g. French fries, fried chicken, fried fish)?' and 'How often do you eat food that is fried at home (exclude "Pam"-type spray)?' Both questions had four possible frequency responses: <1/week, 1-3/week, 4-6/week or daily. We analysed fried food consumption at home and away from home separately, as well as their sum (total fried food consumption). In addition, we asked the participants what kind of frying fat/oils they usually used at home, with the possible responses as follows: real butter, margarine, vegetable oil, vegetable shortening or lard.

Outcome ascertainment The NHS II participants reported incident GDM on each biennial questionnaire through to 2001. In the case of more than one pregnancy lasting longer than 6 months reported within a 2-year questionnaire period, GDM status was attributed to the first pregnancy. In a prior validation study among a subgroup of the NHS II cohort, 94\% of GDM self-reports were confirmed by medical records [13]. In a random sample of parous women without GDM, $83 \%$ reported a glucose screening test during pregnancy and $100 \%$ reported frequent prenatal urine screenings, suggesting a high level of GDM surveillance in this cohort [13].

Covariates assessment Participants reported their height and weight in 1989 and updated their weight on each biennial questionnaire. Self-reported weight was highly correlated with measured weight $(r=0.97)$ in a previous validation study [18] BMI was computed as weight in kilograms divided by height in metres squared. Total physical activity was ascertained by frequency of engaging in common recreational activities, from which metabolic equivalent (MET)-hours per week were derived. The questionnaire-based estimates correlated well with detailed activity diaries in a prior validation study $(r=0.56)$ [19].

To assess the overall diet quality of the participants, we derived a diet score, the Alternate Healthy Eating Index 2010 (AHEI-2010), for each participant, as described previously [20]. Briefly, we allotted 0 to 10 points for intake of each of the following components, with adherence to dietary recommendations receiving 10 points: vegetables (servings/day), fruits (servings/day), whole grains (g/day), sugar-sweetened 
beverages and fruit juice (inversely scored, servings/day), nuts and legumes (servings/day), red/processed meat (inversely scored, servings/day), trans-fat (inversely scored, $\%$ of energy), long-chain $n-3$ fats $(\mathrm{mg} / \mathrm{d})$, polyunsaturated fat $(\%$ of energy), sodium (inversely scored, mg/day) and alcohol (drinks/day; we assigned the highest score to moderate [0.51.5 drinks/day] and the worst score to heavy [ $\geq 2.5$ drinks/day] alcohol consumers). The overall AHEI-2010 ranged from 0 to 110 points, with a higher score indicating a better diet quality and associated with a lower risk of incident chronic diseases, including cardiovascular disease, diabetes and cancer [20].

Statistical analysis Descriptive statistics at baseline were presented as means (SD) for continuous variables and percentages for categorical variables. We used generalised estimating equations (GEE) with log-binomials models [21] to estimate RRs and 95\% CIs. The GEE method allows us to account for correlations among repeated observations (pregnancies) contributed by a single participant [22]. In a few instances, the log-binomials models did not converge, and log-Poisson models [23], which provide consistent but not fully efficient risk estimates, were used.

Pre-pregnancy covariates in the multivariable models included age (continuous), parity $(0,1,2$ and $\geq 3$ pregnancies lasting $\geq 6$ months), race/ethnicity (Caucasian, AfricanAmerican, Hispanic, Asian, other and missing/not reported), family history of diabetes (yes, no), cigarette smoking (never, past, current and missing/not reported), physical activity (quartiles), total energy intake (quartiles) and diet quality as represented by the AHEI-2010 dietary pattern score (quartiles). All these variables, except race/ethnicity and family history of diabetes that were reported in 1989 only, were updated during the follow-up (ESM Fig. 2). In addition, we further adjusted for biennially updated measures of BMI $(<21$, 21-22.9, 23.0-24.9, 25.0-26.9, 27.0-28.9, 29.0-30.9, 31.0$32.9,33.0-34.9, \geq 35.0 \mathrm{~kg} / \mathrm{m}^{2}$ and missing $/$ not reported). BMI was modelled separately because it may also be an intermediate on the pathway between fried food consumption and GDM risk. We derived covariates from the questionnaire preceding each pregnancy. Categorical covariates included an indicator variable for missing data, if necessary. Less than $3 \%$ of covariate data was missing for a given variable. Tests for linear trends were conducted across categories of fried food consumption by assigning the median intake for each category and fitting this continuous variable in the models.

To evaluate effect modification, we performed stratified analyses according to age ( $<35$ vs $\geq 35$ ), parity (nulliparous vs parous), family history of diabetes (yes vs no), prepregnancy physical activity ( $<$ median $v s \geq$ median), prepregnancy overall diet quality (AHEI-2010 dietary pattern score $<$ median vs $\geq$ median) and pre-pregnancy overweight status (BMI $<25 \mathrm{vs} \geq 25 \mathrm{~kg} / \mathrm{m}^{2}$ ). We tested interactions between these factors and fried food consumption by adding multiplicative interaction terms of the binary effect modifiers and continuous linear trend variables in the multivariable models. For fried food consumption at home, we also performed a stratified analysis by types of frying oils.

All statistical analyses were performed with SAS software (version 9.2; SAS Institute, Cary, North Carolina, USA). A $p$ value $<0.05$ was considered statistically significant.

\section{Results}

During the 10 years of follow-up, we documented 847 incident GDM pregnancies among 21,079 eligible singleton pregnancies from 15,027 women. At baseline in 1991, women with higher consumption of fried foods were younger, less likely to be nulliparous and white, and more likely to be current smokers (Table 1). They had a lower diet quality as represented by the AHEI-2010 dietary pattern score, less physical activity and higher BMI.

We observed an elevated risk of GDM in association with higher total fried food consumption (Table 2). After adjustment for age, parity, race/ethnicity, family history of diabetes, cigarette smoking, physical activity, total energy intake and diet quality, the RR (95\% CI) of GDM among women who consumed total fried foods $\geq 7$ times/week was $2.18(1.53,3.09)$ compared with those who consumed it less than once/week ( $p$ for trend $<0.001$ ). After additional adjustment for BMI, the association was moderately attenuated but remained significant, with a corresponding RR $(95 \% \mathrm{CI})$ of $1.88(1.34,2.64)$ ( $p$ for trend $=0.01$ ). Further adjustment for French fried potatoes consumption or trans-fat intake did not substantially alter the results. In addition, the association was not significantly modified by other risk factors of GDM, such as age, parity, family history of diabetes, physical activity, overall diet quality or overweight status (all $p$ for interaction $>0.20$ ).

When analysed separately, we found a significant association of GDM with fried food consumption away from home, but not with fried food consumption at home (Table 3). The multivariable-adjusted RR ( $95 \% \mathrm{CI})$ of GDM comparing consumption of $\geq 4$ times/week with less than once/week of fried food consumption away from home was $1.63(1.15,2.33)$ ( $p$ for trend $<0.001$ ). The association remained significant after additional adjustment for BMI, with a corresponding RR ( $95 \%$ CI) of $1.46(1.03,2.07)$ ( $p$ for trend=0.03). Although women who consumed fried food consumption at home $\geq 4$ times/week seemed to have a $30 \%$ (adjusted RR $1.30,95 \%$ CI $0.81,2.10$ ) higher risk of GDM compared with women who consumed less than once/week, the association was not statistically significant. Additional adjustment for types of frying oils at home did not substantially alter the results. We also conducted a sensitivity analysis by excluding women who were currently pregnant at the time of FFQ and found similar results. 
Table 1 Age-standardised characteristics of the study population $(n=15,027)$ in 1991, according to frequency of total fried food consumption

\begin{tabular}{|c|c|c|c|c|c|}
\hline \multirow[t]{2}{*}{ Characteristic } & \multicolumn{4}{|c|}{ Frequency of total fried food consumption } & \multirow[t]{2}{*}{$p$ value for trend } \\
\hline & $<1 /$ week & $1-3 /$ week & 4-6/week & $\geq 7 /$ week & \\
\hline No. of participants & 7,742 & 4,986 & 2,027 & 272 & - \\
\hline Age, years & $32.3(3.3)$ & $31.8(3.2)$ & $31.4(3.2)$ & $31.1(3.2)$ & $<0.001$ \\
\hline White, $\%$ & 94 & 93 & 91 & 87 & $<0.001$ \\
\hline Family history of diabetes, $\%$ & 11 & 11 & 11 & 16 & 0.11 \\
\hline Nulliparous, \% & 38 & 35 & 33 & 28 & $<0.001$ \\
\hline Current smoker, $\%$ & 8 & 9 & 11 & 12 & $<0.001$ \\
\hline Alcohol, g/day & $3.1(5.1)$ & $3.0(5.2)$ & $2.9(5.6)$ & $2.4(4.5)$ & 0.03 \\
\hline BMI, kg/m² & $23.0(3.9)$ & $23.7(4.5)$ & $24.3(5.0)$ & $24.7(5.7)$ & $<0.001$ \\
\hline Physical activity, MET-h/week & $26.4(31.7)$ & $20.8(25.5)$ & $18.3(24.4)$ & $16.1(22.8)$ & $<0.001$ \\
\hline Total energy intake, $\mathrm{kJ} /$ day & $7,293(2,213)$ & $7,828(2,272)$ & $8,473(2,385)$ & $9,217(2,590)$ & $<0.001$ \\
\hline Carbohydrate, $\% \mathrm{E}$ & $51.9(7.3)$ & $49.8(6.8)$ & $48.4(7.0)$ & $46.3(7.7)$ & $<0.001$ \\
\hline Total protein, $\% \mathrm{E}$ & $19.7(3.4)$ & $18.8(3.1)$ & $18.4(3.0)$ & $18.2(3.3)$ & $<0.001$ \\
\hline Total fat, $\% \mathrm{E}$ & $29.2(5.2)$ & $32.1(4.9)$ & $33.9(5.1)$ & $36.3(5.6)$ & $<0.001$ \\
\hline Saturated fat, $\% \mathrm{E}$ & $10.6(2.3)$ & $11.6(2.2)$ & $12.2(2.3)$ & $13.0(2.5)$ & $<0.001$ \\
\hline Monounsaturated fat, $\% \mathrm{E}$ & $10.8(2.2)$ & $12.2(2.1)$ & $13.1(2.2)$ & $14.4(2.4)$ & $<0.001$ \\
\hline Polyunsaturated fat, $\% \mathrm{E}$ & $5.3(1.3)$ & $5.6(1.3)$ & $5.7(1.3)$ & $5.8(1.2)$ & $<0.001$ \\
\hline trans-Fat, $\% \mathrm{E}$ & $1.3(0.4)$ & $1.8(0.5)$ & $2.0(0.6)$ & $2.5(0.8)$ & $<0.001$ \\
\hline Cholesterol, mg/day ${ }^{\mathrm{a}}$ & $233(66)$ & $239(62)$ & $248(62)$ & $253(67)$ & $<0.001$ \\
\hline Glycaemic index ${ }^{\mathrm{a}}$ & $53.5(3.3)$ & $54.4(3.1)$ & $54.8(3.0)$ & $55.4(2.9)$ & $<0.001$ \\
\hline Glycaemic load $^{\mathrm{a}}$ & $126(21)$ & $122(20)$ & $119(20)$ & $114(22)$ & $<0.001$ \\
\hline Total fibre, $\mathrm{g} / \mathrm{day}^{\mathrm{a}}$ & $19.2(5.8)$ & $17.2(4.8)$ & $16.2(4.4)$ & $14.9(3.9)$ & $<0.001$ \\
\hline Magnesium, mg/day ${ }^{\mathrm{a}}$ & $336(74)$ & $307(68)$ & $288(64)$ & $264(58)$ & $<0.001$ \\
\hline Haem iron, mg/day ${ }^{\mathrm{a}}$ & $1.0(0.4)$ & $1.1(0.4)$ & $1.2(0.4)$ & $1.3(0.5)$ & $<0.001$ \\
\hline Potassium, mg/day ${ }^{\mathrm{a}}$ & $3,013(504)$ & $2,780(468)$ & $2,679(459)$ & $2,558(461)$ & $<0.001$ \\
\hline Calcium, mg/day ${ }^{\mathrm{a}}$ & $1,139(434)$ & $1,034(406)$ & $938(373)$ & $822(352)$ & $<0.001$ \\
\hline Vitamin $\mathrm{C}, \mathrm{mg} /$ day $^{\mathrm{a}}$ & $273(297)$ & $230(250)$ & $201(225)$ & $188(259)$ & $<0.001$ \\
\hline Vitamin E, mg/day ${ }^{\mathrm{a}}$ & $21.6(46.9)$ & $19.7(42.3)$ & $18.3(39.9)$ & $17.2(37.2)$ & 0.04 \\
\hline Red meat, servings/day & $0.6(0.4)$ & $0.8(0.5)$ & $1.1(0.6)$ & $1.5(0.9)$ & $<0.001$ \\
\hline Poultry, servings/day & $0.5(0.3)$ & $0.5(0.3)$ & $0.4(0.3)$ & $0.4(0.3)$ & 0.01 \\
\hline Fish, servings/day & $0.2(0.2)$ & $0.2(0.2)$ & $0.2(0.2)$ & $0.2(0.3)$ & 0.002 \\
\hline Eggs, servings/day & $0.2(0.2)$ & $0.2(0.2)$ & $0.2(0.2)$ & $0.2(0.2)$ & $<0.001$ \\
\hline Low-fat dairy, servings/day & $1.7(1.3)$ & $1.5(1.2)$ & $1.3(1.2)$ & $1.1(1.2)$ & $<0.001$ \\
\hline High-fat dairy, servings/day & $0.9(0.9)$ & $1.0(0.9)$ & $1.1(1.0)$ & $1.1(1.0)$ & $<0.001$ \\
\hline Nuts, servings/day & $0.3(0.4)$ & $0.3(0.3)$ & $0.3(0.3)$ & $0.2(0.2)$ & 0.04 \\
\hline Legumes, servings/day & $0.4(0.3)$ & $0.3(0.3)$ & $0.4(0.3)$ & $0.4(0.3)$ & 0.09 \\
\hline Vegetables, servings/day & $3.4(2.1)$ & $3.0(1.8)$ & $3.0(1.8)$ & $2.8(1.6)$ & $<0.001$ \\
\hline Fruits, servings/day & $1.4(1.0)$ & $1.1(0.9)$ & $1.0(0.8)$ & $0.8(0.7)$ & $<0.001$ \\
\hline Whole grains, servings/day & $1.6(1.2)$ & $1.4(1.1)$ & $1.2(1.0)$ & $1.1(1.0)$ & $<0.001$ \\
\hline SSBs, servings/day & $0.4(0.7)$ & $0.6(0.9)$ & $0.8(1.0)$ & $0.9(1.1)$ & $<0.001$ \\
\hline AHEI-2010 score & $51.7(10.4)$ & $45.8(10.1)$ & $42.3(9.6)$ & $38.2(9.2)$ & $<0.001$ \\
\hline
\end{tabular}

Data are means (SD), unless otherwise specified and are standardised to the age distribution of the study population

Using the conversion factor $1 \mathrm{kcal}=4.184 \mathrm{~kJ}$, total calorie intakes across categories of total fried food consumption were 1,743 (529), 1,871 (543), 2,025 (570), and 2,203 (619) kcal/day

$\mathrm{SSB}$, sugar-sweetened beverage; $\% \mathrm{E}, \%$ of total energy

${ }^{a}$ Indicates values are energy-adjusted 
Table 2 Risk of GDM in association with frequency of pre-pregnancy total fried food consumption

\begin{tabular}{|c|c|c|c|c|c|}
\hline & \multicolumn{4}{|c|}{ Frequency of total fried food consumption } & \multirow[t]{2}{*}{$p$ value for trend } \\
\hline & $<1 /$ week & $1-3 /$ week & 4-6/week & $\geq 7 /$ week & \\
\hline GDM/pregnancies & $355 / 10,518$ & $304 / 7,181$ & $156 / 3,032$ & $32 / 348$ & \\
\hline Model 1 & 1.00 (reference) & $1.28(1.10,1.49)$ & $1.60(1.33,1.92)$ & $3.07(2.19,4.30)$ & $<0.001$ \\
\hline Model 2 & 1.00 (reference) & $1.23(1.06,1.43)$ & $1.49(1.24,1.79)$ & $2.55(1.81,3.59)$ & $<0.001$ \\
\hline Model 3 & 1.00 (reference) & $1.13(0.97,1.32)$ & $1.31(1.08,1.59)$ & $2.18(1.53,3.09)$ & $<0.001$ \\
\hline Model 4 & 1.00 (reference) & $1.06(0.91,1.23)$ & $1.14(0.94,1.38)$ & $1.88(1.34,2.64)$ & 0.01 \\
\hline
\end{tabular}

Data are shown as RRs $(95 \% \mathrm{CI})$

Model 1: age (months) and parity ( $0,1,2$ and $\geq 3$ pregnancies lasting $\geq 6$ months)

Model 2: Model $1+$ race/ethnicity (Caucasian, African-American, Hispanic, Asian, others and missing/not reported), family history of diabetes (yes, no), cigarette smoking (never, past, current and missing/not reported) and physical activity (quartiles)

Model 3: Model $2+$ total energy intake (quartiles) and diet quality (i.e. AHEI-2010 score, quartiles)

Model 4: Model $3+$ BMI $\left(<21,21-22.9,23.0-24.9,25.0-26.9,27.0-28.9,29.0-30.9,31.0-32.9,33.0-34.9\right.$ and $\left.\geq 35.0 \mathrm{~kg} / \mathrm{m}^{2}\right)$

In our cohort, women with higher consumption of fried food consumed more red meat and sugar-sweetened beverages. Although these dietary factors were components of the AHEI-2010 dietary pattern score, we conducted a sensitivity analysis further adjusting for red meat and sugar-sweetened beverages, which slightly attenuated the associations. The multivariable-adjusted RRs (95\% CIs) of GDM among women who consumed total fried foods $1-3,4-6$ and $\geq 7$ times/ week, compared with those who consumed less than once/week, were $1.04(0.89,1.21), 1.09(0.90,1.32)$ and $1.78(1.27,2.51)$, respectively ( $p$ for trend $=0.04$ ).

\section{Discussion}

In this large prospective cohort study, we found a significant and positive association between pre-pregnancy fried food consumption and the risk of GDM. The association was

Table 3 Risk of GDM in association with frequency of pre-pregnancy fried food consumption at home and away from home

\begin{tabular}{|c|c|c|c|c|}
\hline & \multicolumn{3}{|c|}{ Frequency of fried food consumption } & \multirow[t]{2}{*}{$p$ value for trend } \\
\hline & $<1 /$ week & $1-3 /$ week & $\geq 4 /$ week & \\
\hline \multicolumn{5}{|l|}{ At home } \\
\hline GDM/pregnancies & $554 / 15,042$ & $276 / 5,773$ & $17 / 264$ & \\
\hline Model 1 & 1.00 (reference) & $1.19(1.03,1.38)$ & $1.55(0.98,2.46)$ & 0.004 \\
\hline Model 2 & 1.00 (reference) & $1.18(1.02,1.36)$ & $1.37(0.86,2.20)$ & 0.01 \\
\hline Model 3 & 1.00 (reference) & $1.14(0.98,1.31)$ & $1.30(0.81,2.10)$ & 0.054 \\
\hline Model 4 & 1.00 (reference) & $1.07(0.92,1.23)$ & $1.37(0.88,2.13)$ & 0.18 \\
\hline \multicolumn{5}{|l|}{ Away from home } \\
\hline GDM/pregnancies & $475 / 13,493$ & $341 / 7,170$ & $31 / 416$ & \\
\hline Model 1 & 1.00 (reference) & $1.40(1.22,1.61)$ & $2.15(1.52,3.05)$ & $<0.001$ \\
\hline Model 2 & 1.00 (reference) & $1.32(1.15,1.52)$ & $1.87(1.32,2.66)$ & $<0.001$ \\
\hline Model 3 & 1.00 (reference) & $1.21(1.05,1.40)$ & $1.63(1.15,2.33)$ & $<0.001$ \\
\hline Model 4 & 1.00 (reference) & $1.11(0.96,1.28)$ & $1.46(1.03,2.07)$ & 0.03 \\
\hline
\end{tabular}

Data are shown as RRs $(95 \%$ CIs $)$

Model 1: age (months) and parity ( $0,1,2$ and $\geq 3$ pregnancies lasting $\geq 6$ months)

Model 2: Model $1+$ race/ethnicity (Caucasian, African-American, Hispanic, Asian, others and missing/not reported), family history of diabetes (yes, no), cigarette smoking (never, past, current and missing/not reported) and physical activity (quartiles)

Model 3: Model $2+$ total energy intake (quartiles) and diet quality (i.e. AHEI-2010 score, quartiles)

Model 4: Model 3 + BMI $\left(<21,21-22.9,23.0-24.9,25.0-26.9,27.0-28.9,29.0-30.9,31.0-32.9,33.0-34.9\right.$ and $\left.\geq 35.0 \mathrm{~kg} / \mathrm{m}^{2}\right)$ 
attenuated, but remained significant, after additional adjustment for dietary and non-dietary factors, including BMI. In addition, the association was not significantly modified by age, parity, family history of diabetes, physical activity, overall diet quality or overweight status. Fried food consumed away from home may have a stronger association with GDM risk than fried food consumed at home.

To our knowledge, the association between fried food consumption and risk of GDM has not been previously examined. There is evidence, although still limited, indicating that frequent fried food consumption is associated with increased risk of obesity and type 2 diabetes among non-pregnant individuals. During a median of 6.1 years of follow-up in the SUN project (a Mediterranean cohort), participants who consumed fried foods more than four times/week had a $37 \%$ higher risk of developing overweight/obesity in comparison with those who consumed fried foods less than twice/week [7]. Similarly, fried food consumption was also positively associated with general and central obesity in a cross-sectional analysis in another Mediterranean cohort [6]. In a prospective cohort study of African-American women, frequent fried chicken consumption was associated with risk of incident type 2 diabetes [24]. Recently, an analysis of two large prospective cohorts among US men and women also reported that frequent fried food consumption was significantly associated with an increased risk of incident type 2 diabetes [25].

Frequent fried food consumption could be an indicator of unhealthy dietary habits. In our study population, we observed that women who consumed fried foods more frequently had greater total energy intake and poorer diet quality, which is consistent with findings from a previous study [26]. However, the association between fried food consumption and GDM risk persisted after adjustment for these dietary variables. Moreover, additional adjustment for specific food groups (i.e. red meat, processed meat) did not materially alter the observed association.

The potential detrimental effects of fried food consumption on GDM risk may result from the modification of foods and frying medium, and generation of harmful by-products during the frying process. Frying deteriorates oils through the processes of oxidation and hydrogenation [5], leading to an increase in the absorption of oil degradation products by the foods being fried, and also a loss of unsaturated fatty acids such as linoleic and linolenic acids and an increase in the corresponding trans-fatty acids such as trans-linoleic acids and trans-linolenic acids [27]. Several epidemiological studies and experimental studies have linked higher intake of transfatty acids to reduced insulin sensitivity and increased risk of type 2 diabetes [28]. There was suggestive evidence showing that higher intake of trans-fat may be associated with greater risk of incident GDM, although the association was no longer significant after additional adjustment for other types of dietary fats [29]. In the present study, additional adjustment of trans-fat intake did not appreciably alter the association between fried food consumption and GDM, indicating that the association cannot be fully explained by trans-fat. Frying also results in significantly higher levels of dietary AGEs, the derivatives of glucose-protein or glucose-lipid interactions [30]. Recently, AGEs have been implicated in insulin resistance, pancreatic beta cell damage and diabetes, partly because they promote oxidative stress and inflammation [31, 32]. Moreover, intervention studies with a diet low in AGEs have shown significantly improved insulin sensitivity [33], reduced oxidant stress and alleviated inflammation [34]. The above-mentioned deterioration of oils during frying is more profound when the oils are reused [35], a practice more common away from home than at home. This may partly explain why we observed a stronger association of GDM risk with fried foods consumed away from home than fried foods consumed at home.

Our study has several strengths, including the prospective design that establishes the temporal direction of the associations, the large sample size and the repeated comprehensive assessment of many lifestyle characteristics that were collected prospectively with a long duration of follow-up. Therefore, we were able to assess the confounding effects and potential modification of the association for fried food consumption by other dietary components or lifestyle factors. We acknowledge that there are several limitations. First, we did not have information about the specific types (e.g. beef, chicken, fish or vegetables, etc.), serving size or frying methods (e.g. deep or pan frying; fresh or reused oil; duration and temperature; types of oil [i.e. olive, corn, vegetable, etc.] used away from home, etc.) of fried foods consumed by our participants. Second, our study population consisted mostly of white American women. Thus, the ability to directly generalise the observed associations may be limited to similar populations. However, the relative homogeneity of the study population reduces potential confounding owing to unmeasured socioeconomic variability.

In conclusion, we observed that frequent fried food consumption was significantly and positively associated with the risk of incident GDM in a prospective cohort study. Our study indicates potential benefits of limiting fried food consumption in the prevention of GDM in women of reproductive age. Further studies are warranted to confirm our findings and to elucidate the underlying mechanisms.

Funding This study was supported by the Intramural Research Program of the Eunice Kennedy Shriver National Institute of Child Health and Human Development, National Institutes of Health (contract No. HHSN275201000020C). The Nurses' Health Study II was funded by research grants DK58845, CA50385, P30 DK46200 and UM1 CA176726 from the National Institutes of Health. DKT was supported by a mentored fellowship from the American Diabetes Association (No. 7-12-MN-34).

Duality of interest All authors declare that there is no duality of interest associated with this manuscript. 
Contribution statement WB contributed to the design and analysis of the study and wrote the manuscript. DKT interpreted the data, conducted technique review and reviewed and edited the manuscript. SFO interpreted the data and reviewed and edited the manuscript. CZ contributed to the design and analysis of the study, interpreted the data and reviewed and edited the manuscript. All authors approved the final version. WB and $\mathrm{CZ}$ are the guarantors of this work and, as such, had full access to all the data in the study and take responsibility for the integrity of the data and the accuracy of the data analysis.

\section{References}

1. Iqbal R, Anand S, Ounpuu S et al (2008) Dietary patterns and the risk of acute myocardial infarction in 52 countries: results of the INTERHEART study. Circulation 118:1929-1937

2. Hu FB (2008) Globalization of food patterns and cardiovascular disease risk. Circulation 118:1913-1914

3. Popkin BM (2006) Global nutrition dynamics: the world is shifting rapidly toward a diet linked with noncommunicable diseases. Am J Clin Nutr 84:289-298

4. Pan A, Malik VS, Hu FB (2012) Exporting diabetes mellitus to Asia: the impact of western-style fast food. Circulation 126:163-165

5. Guallar-Castillon P, Rodriguez-Artalejo F, Lopez-Garcia E et al (2012) Consumption of fried foods and risk of coronary heart disease: Spanish cohort of the European Prospective Investigation into Cancer and Nutrition study. BMJ 344:e363

6. Guallar-Castillon P, Rodriguez-Artalejo F, Fornes NS et al (2007) Intake of fried foods is associated with obesity in the cohort of Spanish adults from the European Prospective Investigation into Cancer and Nutrition. Am J Clin Nutr 86:198-205

7. Sayon-Orea C, Bes-Rastrollo M, Basterra-Gortari FJ et al (2013) Consumption of fried foods and weight gain in a Mediterranean cohort: the SUN project. Nutr Metab Cardiovasc Dis 23:144-150

8. American Diabetes Association (2004) Gestational diabetes mellitus. Diabetes Care 27(Suppl 1):S88-S90

9. Metzger BE, Lowe LP, Dyer AR et al (2008) Hyperglycemia and adverse pregnancy outcomes. N Engl J Med 358:1991-2002

10. Reece EA, Leguizamon G, Wiznitzer A (2009) Gestational diabetes: the need for a common ground. Lancet 373:1789-1797

11. Bellamy L, Casas JP, Hingorani AD, Williams D (2009) Type 2 diabetes mellitus after gestational diabetes: a systematic review and meta-analysis. Lancet 373:1773-1779

12. Zhang C, Ning Y (2011) Effect of dietary and lifestyle factors on the risk of gestational diabetes: review of epidemiologic evidence. Am J Clin Nutr 94:1975S-1979S

13. Solomon CG, Willett WC, Carey VJ et al (1997) A prospective study of pregravid determinants of gestational diabetes mellitus. JAMA 278:1078-1083

14. Colditz GA, Hankinson SE (2005) The Nurses' Health Study: lifestyle and health among women. Nat Rev Cancer 5:388-396

15. Willett WC, Sampson L, Stampfer MJ et al (1985) Reproducibility and validity of a semiquantitative food frequency questionnaire. Am J Epidemiol 122:51-65

16. Willett WC, Sampson L, Browne ML et al (1988) The use of a selfadministered questionnaire to assess diet four years in the past. Am J Epidemiol 127:188-199
17. Salvini S, Hunter DJ, Sampson L et al (1989) Food-based validation of a dietary questionnaire: the effects of week-to-week variation in food consumption. Int J Epidemiol 18:858-867

18. Rimm EB, Stampfer MJ, Colditz GA, Chute CG, Litin LB, Willett WC (1990) Validity of self-reported waist and hip circumferences in men and women. Epidemiology 1:466-473

19. Wolf AM, Hunter DJ, Colditz GA et al (1994) Reproducibility and validity of a self-administered physical activity questionnaire. Int J Epidemiol 23:991-999

20. Chiuve SE, Fung TT, Rimm EB et al (2012) Alternative dietary indices both strongly predict risk of chronic disease. J Nutr 142: 1009-1018

21. Wacholder S (1986) Binomial regression in GLIM: estimating risk ratios and risk differences. Am J Epidemiol 123:174-184

22. Hanley JA, Negassa A, Edwardes MD, Forrester JE (2003) Statistical analysis of correlated data using generalized estimating equations: an orientation. Am J Epidemiol 157:364-375

23. Zou G (2004) A modified poisson regression approach to prospective studies with binary data. Am J Epidemiol 159:702-706

24. Krishnan S, Coogan PF, Boggs DA, Rosenberg L, Palmer JR (2010) Consumption of restaurant foods and incidence of type 2 diabetes in African American women. Am J Clin Nutr 91:465-471

25. Cahill LE, Pan A, Chiuve SE et al (2014) Fried-food consumption and risk of type 2 diabetes and coronary artery disease: a prospective study in 2 cohorts of US women and men. Am J Clin Nutr 100:667675

26. Taveras EM, Berkey CS, Rifas-Shiman SL et al (2005) Association of consumption of fried food away from home with body mass index and diet quality in older children and adolescents. Pediatrics 116: e518-e524

27. Li A, Ha Y, Wang F, Li W, Li Q (2012) Determination of thermally induced trans-fatty acids in soybean oil by attenuated total reflectance Fourier transform infrared spectroscopy and gas chromatography analysis. J Agric Food Chem 60:10709-10713

28. Mozaffarian D, Aro A, Willett WC (2009) Health effects of transfatty acids: experimental and observational evidence. Eur J Clin Nutr 63(Suppl 2):S5-S21

29. Bowers K, Tobias DK, Yeung E, Hu FB, Zhang C (2012) A prospective study of prepregnancy dietary fat intake and risk of gestational diabetes. Am J Clin Nutr 95:446-453

30. Goldberg T, Cai W, Peppa M et al (2004) Advanced glycoxidation end products in commonly consumed foods. J Am Diet Assoc 104: 1287-1291

31. Cai W, Ramdas M, Zhu L, Chen X, Striker GE, Vlassara H (2012) Oral advanced glycation endproducts (AGEs) promote insulin resistance and diabetes by depleting the antioxidant defenses AGE receptor-1 and sirtuin 1. Proc Natl Acad Sci U S A 109:15888-15893

32. Coughlan MT, Yap FY, Tong DC et al (2011) Advanced glycation end products are direct modulators of beta-cell function. Diabetes 60: 2523-2532

33. Mark AB, Poulsen MW, Andersen S et al (2014) Consumption of a diet low in advanced glycation endproducts for 4 weeks improves insulin sensitivity in overweight women. Diabetes Care 37:88-95

34. Vlassara H, Cai W, Goodman S et al (2009) Protection against loss of innate defenses in adulthood by low advanced glycation end products (AGE) intake: role of the antiinflammatory AGE receptor-1. J Clin Endocrinol Metab 94:4483-4491

35. Lee WT, Dawson LE (1973) Chicken lipid changes during cooking in fresh and reused cooking oil. J Food Sci 38:1232-1237 